\title{
Education and gastroenterology fellow knowledge about endoscopic mucosal resection of colon adenomas: a survey-based study
}

다(1)우우

\author{
Authors \\ Institutions \\ 1 Division of Gastroenterology Department of Medicine, \\ UAMS, Little Rock, Arkansas, United States \\ 2 Division of Gastroenterology, Department of Medicine, \\ Yale School of Medicine, New Haven, Connecticut, \\ United States
}

Shashank Garg1 , Sumant Inamdar ${ }^{1}$, Benjamin Tharian' ${ }^{1}$, Thiruvengadam Muniraj², Harry R. Aslanian²

submitted 17.11 .2020

accepted after revision 26.3.2021

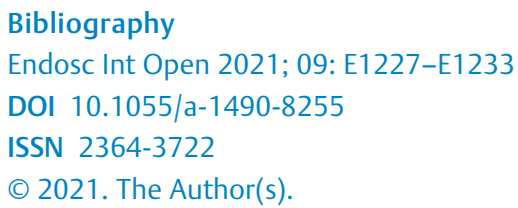
Commons Attribution-NonDerivative-NonCommercial License, permitting copying and reproduction so long as the original work is given appropriate credit. Contents may not be used for commercial purposes, or adapted, remixed, transformed or built upon. (https://creativecommons.org/licenses/by-nc-nd/4.0/)

Georg Thieme Verlag KG, Rüdigerstraße 14,

70469 Stuttgart, Germany

Corresponding author

Shashank Garg, MD, MS, 4301 W. Markham St., Suite S8/68, Little Rock, AR 72223, United States

Fax: +1-501-686-6248

sgarg@uams.edu

Supplementary material is available under https://doi.org/10.1055/a-1490-8255

\section{ABSTRACT}

Background and study aims Endoscopic mucosal resection (EMR) is an effective way to remove large $(>2 \mathrm{~cm})$ colon adenomas. Training about it has not been standardized in fellowship programs. This study was aimed at evaluating the education and knowledge of gastroenterology fellows about EMR of colorectal adenomas.

Methods Participation in this survey was offered to 1730 gastroenterology fellows in the United States during the academic year 2019 to 2020 . The survey assessed endoscopic mucosal resection training and knowledge and was approved and administered by the American College of Gastroenterology.

Results A total of 163 fellows (9.4\%) completed the survey. Only 85 fellows $(52.1 \%$ ) reported receiving formal education in endoscopic mucosal resection. Fellow confidence was lowest regarding knowing electrosurgery unit settings. Fewer fellows correctly identified Paris 0-IIb (79, 48.5\%; $P<$ $0.0001)$ or NICE I $(114,69.9 \%$; $P<0.01)$ lesions as compared to other Paris and NICE III lesions, respectively. Only 73 (44.8\%) and 93 fellows (57.1\%) arranged steps of EMR in the correct order and identified the correct type of current used for resection, respectively. Training year, male sex, and provision of advanced endoscopy rotations during fellowship were associated with a higher knowledge score for EMR.

Conclusions Nearly half of all fellows reported no formal education in EMR and incorrectly ordered its steps. Adenoma assessment by Paris and NICE classifications and electrosurgery unit settings were the most prominent knowledge deficiencies. Incorporation of standardized training about EMR with inclusion of advanced endoscopy rotations appears to be an important educational opportunity during gastroenterology fellowship.

\section{Introduction}

Colorectal cancer (CRC) is the third most common cancer and the third leading cause of cancer-related death in both men and women in the United States [1]. CRC develops from adenomas and adenomas $>1 \mathrm{~cm}$ progress to CRC more frequently and in a shorter time as compared to smaller adenomas $(<1 \mathrm{~cm})[2]$. Therefore, colonoscopic detection and removal of advanced adenomas is vital for CRC prevention [3-6]. Endoscopic mucosal resection (EMR) techniques foster safe and complete removal of large adenomas $(>2 \mathrm{~cm})$ as compared to conventional snare polypectomy [7]. EMR is a multistep process that requires 
education and practice. Training in EMR has not been standardized among fellowship programs. We are not aware of prior studies that have evaluated the education and knowledge of gastroenterology fellows in endoscopic assessment and resection of large colorectal adenomas. The aims of this survey study were to: (1) evaluate the education and knowledge of resection of large adenomas by EMR and (2) evaluate factors predictive of knowledge of EMR among gastroenterology fellows in the United States.

\section{Methods}

\section{Study population}

We conducted a cross-sectional survey of all gastroenterology fellows in training in the United States during the 2019-2020 academic year. We invited 1730 fellows in 203 fellowship programs to participate after excluding the advanced endoscopy fellows. The study was approved as exempt research by the Institutional Review Board at the University of Arkansas for Medical Sciences.

\section{Survey creation and content}

We developed a five-part, 34-item, multiple-choice question survey that was approved by the research committee of the American College of Gastroenterology (ACG) The first part of the survey was designed to obtain demographic and program information without collecting any identifying information. Parts two to four of the survey were designed to assess the level of education fellows had received in their program (item 14: 14 points) and their confidence in various aspects of EMR (Item 15: 1 points; Supplementary file 1). Part 5 assessed their knowledge of adenoma assessment with the Paris and Narrow-Band Imaging International Colorectal Endoscopic (NICE) classifications and familiarity of details of EMR technique (items 16-34, 19 points). The survey items were designed based on the EMR guidelines issued by the Amercian Society of Gastrointestinal Endoscopy (ASGE) and European Society of Gastrointestinal Endoscopy (ESGE) and were intended to be non-ambiguous [7, 8]. The full survey can be found in Supplement 1.

\section{Survey distribution}

The first email invitation was sent to the fellows by ACG in December 2019 with two additional reminders sent before January 2020. Two email requests were sent to non-responders via fellowship coordinators before the study closed in June 2020 . There was no direct contact between the participants and investigators of the study. Participation in the survey was voluntary and no incentives were paid. Survey creation and data collection were done using Google Forms (Google LLC, Delaware, United States).

\section{Definitions}

A five-point scale was used to assess fellow confidence about different aspects of EMR of colon adenomas. The scale was defined as follows: 1.1 am not familiar with this topic; 2 . Not confident at all e.g. attending does most of the assessment and procedure; 3. Somewhat confident e.g. attending takes the scope often; 4. Confident e.g. attending takes the scope in difficult scenarios; and 5 . Very confident e.g. attending rarely takes the scope.

\section{Data analysis}

Descriptive statistics were used to perform exploratory analyses. Categorical data were described as proportions and analyzed using chi-square test. Continuous data were reported as mean and standard deviation or median and range and analyzed using t-test or Wilcoxon ran-sum test depending on the distribution of the variable. Median confidence was calculated for each fellow based on their responses to the 11 points in item 15. A composite score of education (out of 14 points of item 14) and knowledge (out of 19 points for items 16 to 34) were calculated for all the fellows. Distribution of the total knowledge score (dependent variable) was not normal and therefore, linear regression was not possible. Knowledge-score tertiles were created based on the total knowledge score of the participants. Ordinal regression was done to identify factors associated with knowledge-score tertiles. The area under the curve (AUC) was used to assess model fit. Independent variables included were training year, sex, setting of the program (University, Community or both), size of the program, number of total faculty and advanced faculty in the program, availability of advanced fellowship in the gastroenterology division, provision of advanced endoscopy rotation during fellowship, whether the candidates intended to apply to advanced endoscopy fellowship, physicians performing EMR (general gastroenterology, advanced endoscopist or both), number of EMR cases done by participating fellows during the fellowship, education score, and median confidence. Two-sided $P<0.05$ was considered significant. The analysis was performed with SAS software version 9.4 (SAS Institute Inc., North Carolina, United States).

\section{Results}

\section{Sample characteristics}

The survey response rate was 9.4\% (163 out of 1730). > Table 1 summarizes the demographics, program information, and provision of formal education of EMR in the training programs. The majority of respondents were men $(117,71.8 \%)$, were training in a university setting $(129,79.1 \%)$, and were second$(62,38 \%)$ or third-year fellows $(82,50.3 \%)$. Fellows from all sizes of training programs participated in the survey ( $\triangleright$ Table 1 ). The majority $(102 ; 62.6 \%)$ of the participants reported having $>10$ faculty members involved in their training. All respondents reported having advanced endoscopy faculty in their program ( Table 1$)$. Eighty-two participants $(50.3 \%$ ) reported having an advanced endoscopy fellowship in their division. One hundred three (63.2\%) fellows reported having advanced endoscopy rotation(s) as a part of their training. Most respondents (114, $69.9 \%)$ did not plan on doing an advanced endoscopy fellowship. One hundred and three (63.2\%) fellows reported that EMR of colon adenomas is performed by both general gastroenterology and advanced endoscopy faculty in their program while $31.3 \%$ of fellows $(51 / 163)$ reported that only advanced endoscopists performed EMR. Most respondents (104, 63.8\%) 
- Table 1 Participant and program information.

\begin{tabular}{|c|c|}
\hline Variable & N (\%) \\
\hline \multicolumn{2}{|l|}{ Year in-training } \\
\hline - 1st year fellow & $19(11.7)$ \\
\hline - 2nd year fellow & $62(38)$ \\
\hline - 3 rd year fellow & $82(50.3)$ \\
\hline \multicolumn{2}{|l|}{ Sex } \\
\hline - Male & $117(71.8)$ \\
\hline - Female & $44(27 \%)$ \\
\hline - Did not disclose & $2(1.2)$ \\
\hline \multicolumn{2}{|l|}{ Program setting } \\
\hline - University & $105(64.4)$ \\
\hline - Community & $34(20.9)$ \\
\hline - Both & $24(14.7)$ \\
\hline \multicolumn{2}{|l|}{ Fellows per year } \\
\hline . $1-2$ & $46(28.2)$ \\
\hline . $3-4$ & $64(39.3)$ \\
\hline.$>4$ & $53(32.5)$ \\
\hline \multicolumn{2}{|l|}{ Clinical faculty in program } \\
\hline . $\leq 5$ & $17(10.4)$ \\
\hline . 6-10 & $44(27)$ \\
\hline . $>10$ & $102(62.6)$ \\
\hline \multicolumn{2}{|l|}{ Advanced endoscopy faculty in program } \\
\hline . $1-2$ & $63(38.7)$ \\
\hline . $3-4$ & $46(28.2)$ \\
\hline . $>4$ & $54(33.1)$ \\
\hline $\begin{array}{l}\text { Advanced endoscopy fellowship available in the } \\
\text { division }\end{array}$ & $82(50.3)$ \\
\hline $\begin{array}{l}\text { Advanced endoscopy rotations during gastroen- } \\
\text { terology fellowship }\end{array}$ & $103(63.2)$ \\
\hline $\begin{array}{l}\text { Hands- on experience during advanced endos- } \\
\text { copy rotation }\end{array}$ & 82 of 103 (79.6) \\
\hline $\begin{array}{l}\text { Planning to apply for advanced endoscopy fel- } \\
\text { lowship }\end{array}$ & $49(30.1)$ \\
\hline \multicolumn{2}{|l|}{ Faculty performing large $(>2 \mathrm{~cm})$ colon polyp EMR } \\
\hline - General gastroenterology & $9(5.5)$ \\
\hline - Advanced endoscopist & $51(31.3)$ \\
\hline - Both & $103(3.2)$ \\
\hline \multicolumn{2}{|l|}{ No. of large EMR cases done during fellowship } \\
\hline . $<10$ & $104(63.8)$ \\
\hline . $10-20$ & $42(25.8)$ \\
\hline$\cdot>20$ & $17(10.4)$ \\
\hline $\begin{array}{l}\text { Formal education about EMR provided in pro- } \\
\text { gram }\end{array}$ & $85(52.1)$ \\
\hline
\end{tabular}

- Table 1 (Continuation)

\begin{tabular}{|l|c|}
\hline Variable & N (\%) \\
\hline $\begin{array}{l}\text { EMR training received outside of training pro- } \\
\text { gram }\end{array}$ & $50(30.7)$ \\
\hline Location of such training & \\
\hline - Endoscopy course & 37 of $50(74)$ \\
\hline - Online & 10 of $50(20)$ \\
\hline - Other institution & 3 of $50(6)$ \\
\hline
\end{tabular}

reported that they had participated in $<10$ cases of EMR of large colon adenomas and only $10.4 \%$ of fellows (17/163) reported having done $>20$ cases.

\section{Education provided by fellowship programs for EMR and fellow confidence in performing EMR}

Seventy-eight fellows (47.9\%) reported that they had not received any formal education or training in EMR. Specific areas of EMR with the lowest degree of formal education included text-documentation of EMR $(74,45.4 \%)$, photo documentation of EMR $(85,52.1 \%)$, electrosurgery unit settings for EMR (87, $53.4 \%$ ), assessing pit pattern, surface and borders (91, 55.8\%), when not to attempt $\operatorname{EMR}(98,60.1 \%)$, equipment needed for EMR $(100,61.3 \%)$, and identification and management of complications of EMR $(101,62 \%)$. Areas of EMR with the highest reported degree of formal education included tattooing (137, $84 \%)$, adenoma resection $(135,82.8 \%)$, and assessment of adenoma size and morphology (123, 75.5\%; - Fig. 1). Fifty (30.7\%) fellows reported receiving EMR training outside of their institution ( $\triangleright$ Table 1 ). Reported median confidence was highest for assessing adenoma size and shape and knowing the follow-up colonoscopy interval after EMR. It was lowest for knowing electrosurgery unit settings ( $\triangleright$ Fig. 2 ).

\section{Fellow knowledge of EMR: Endoscopic assessment of adenomas}

More fellows were able to correctly match a pedunculated lesion $(138,84.7 \%)$ to the appropriate Paris class as compared to a sessile raised lesion $(117,71.8 \% ; P=0.01)$. Correct Paris classification of a flat lesion $(79,48.5 \%)$ was far less accurately performed relative to either pedunculated or sessile lesions $(P<$ $0.0001)$. Correct identification of NICE II (129, 79.1\%) and NICE III $(138,84.7 \%)$ lesions was similar among the participants $(P=$ $0.23)$. Correct identification of a NICE I lesion $(114,69.9 \%)$ was lower than NICE III lesions $(P<0.01)$ but similar to NICE II lesions $(P=0.08)$.

\section{Fellow knowledge of EMR: Technical aspects of EMR}

Fewer participants arranged the six basic steps of EMR in the correct order $(73,44.8 \%)$, identified the correct type of current used for $\operatorname{EMR}(93,57.1 \%)$, and correctly identified the most appropriate management strategy for residual polyp tissue at the polypectomy site $(117,71.8 \%)$ as compared to knowing about 


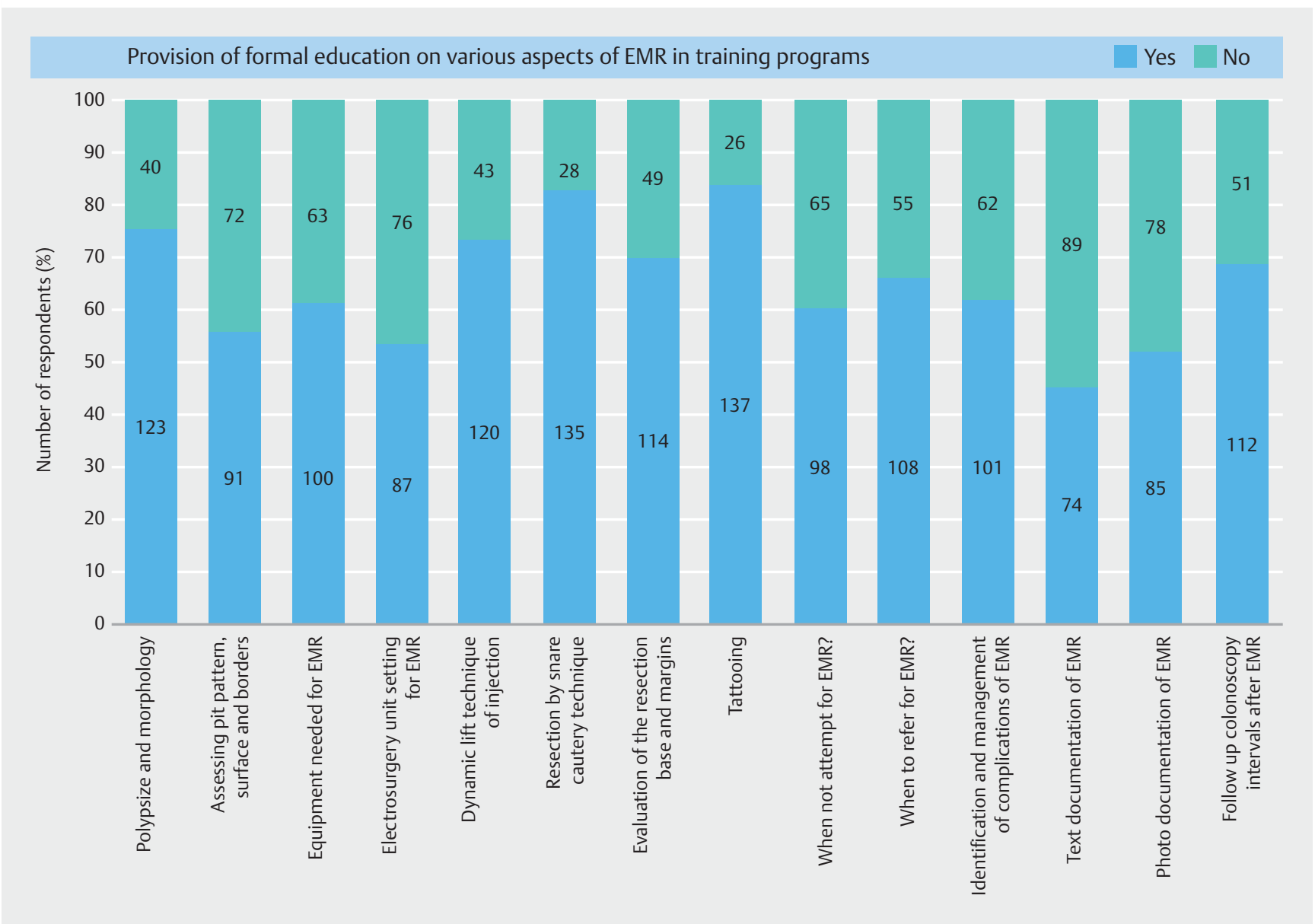

Fig. 1 Formal training given by the program in individual steps of EMR of large colon polyps. EMR, endoscopic mucosal resection.

higher recurrence of adenoma with piecemeal resection (158, 96.9\%; $P<0.0001$; Table 2).

\section{Fellow knowledge of EMR: Technical aspects of EMR: Complications and follow-up}

A lower number of participants correctly identified the most common immediate complication of EMR (121, 74.2\%) and the correct type of current for management of post-polypectomy bleeding $(121,74.2 \%)$ as compared to identifying delayed complications of EMR (147, 90.2\%; $P<0.001)$. Lastly, the majority of the participants $(153,93.9 \%)$ correctly identified the follow-up interval after resection of large adenomas.

\section{Factors associated with fellow knowledge of EMR}

Ordinal regression showed that progression through each year of fellowship was associated with a higher knowledge-score tertile ( $>$ Table 3). Similarly, male sex (OR 3.14, 95\% Cl: 1.48-6.68; $P=0.01)$ and availability of advanced endoscopy rotations were associated with a higher knowledge-score tertile (OR 2.25, $95 \%$ Cl: 1.14-4.44; $P=0.02$; Table 3). Increase in median confidence by each point was associated with lower knowledge score-tertiles (OR 0.67, $95 \% \mathrm{Cl}: 0.47-0.96 ; P=0.03$ ). The other previously listed nine factors, including program size, setting, size of the faculty and education-score, did not have any association with knowledge-score tertiles.

\section{Discussion}

While much attention and study has been dedicated to colonoscopy quality measures such as cecal intubation rate, withdrawal time, and ADR, large adenoma resection, which is the key step in providing colon cancer risk reduction, has received much less attention. This survey study is the first of its kind to evaluate the knowledge of gastroenterology fellows in the United States regarding adenoma classification and EMR techniques. We found that nearly half of all fellows reported no formal education in EMR in their gastroenterology fellowship (48\%) and this response persisted among third year fellows (42.68\%). A possible explanation for this observation is that not all clinical faculty perform EMR; therefore, fellows may not get significant exposure to these procedures. This is supported by the finding that $31.3 \%$ of fellows reported that only advanced endoscopy faculty perform EMR at their center and that $63.8 \%$ of the fellows had participated in $<10$ EMR cases. Additional contributing factors likely include EMR being considered an advanced endoscopy skill that requires further training and that the fellowship programs may provide formal educa- 


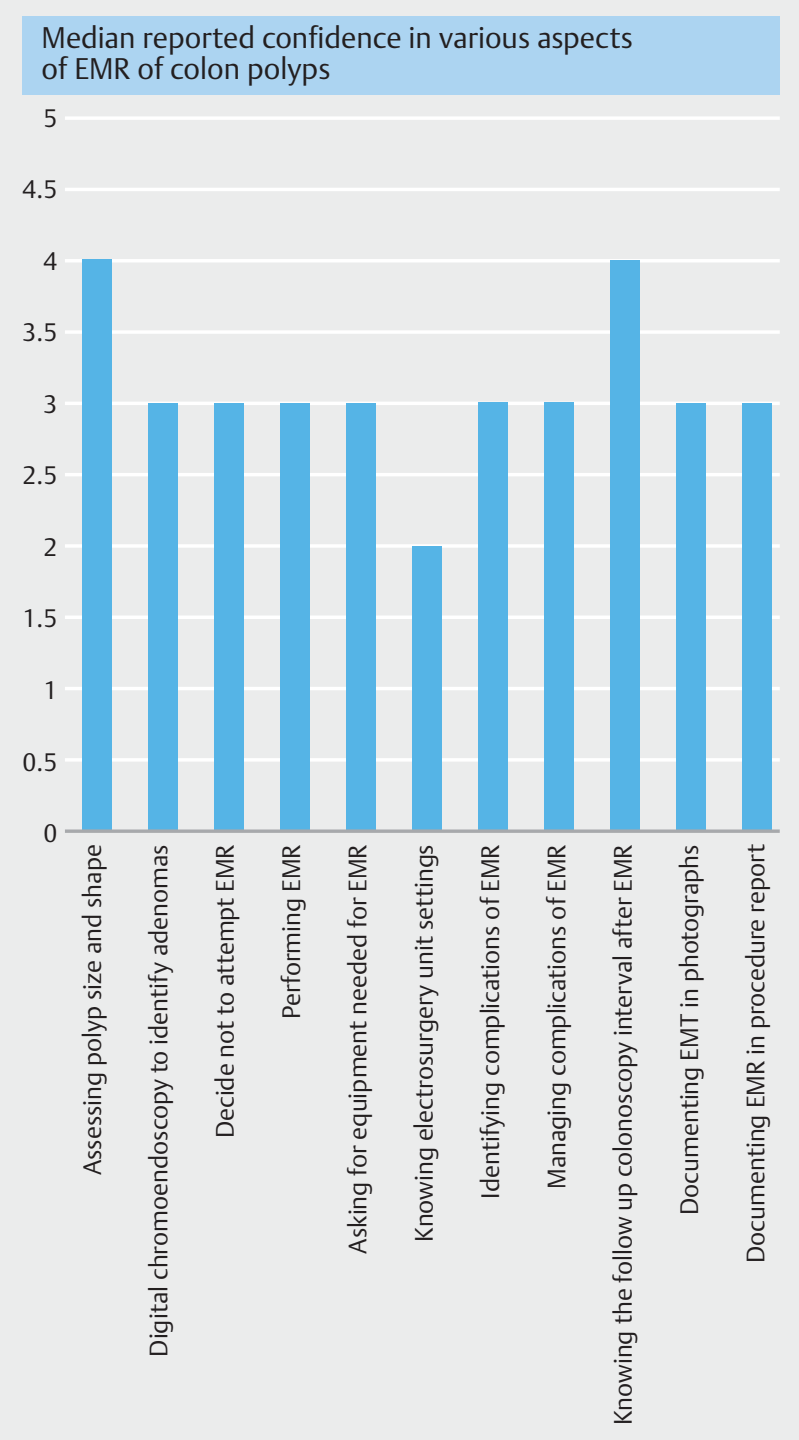

- Fig. 2 Median confidence reported by fellows in individual steps of EMR of large colon polyps. Five-point scale rating: 1.1 am not familiar with this topic; 2 . Not confident at all e. g. attending does most of the assessment and procedure; 3 . Somewhat confident e. g. attending takes the scope often; 4. Confident e.g. attending takes the scope in difficult scenarios; and 5. Very confident e.g. attending rarely takes the scope. EMR: Endoscopic mucosal resection.

tion in various aspects of colonoscopy, but may lack a structured curriculum for the performance of EMR. Further studies are warranted to evaluate the inclusion of EMR training in gastroenterology fellowship curriculum and the impact on knowledge base and clinical outcomes of polyp detection and complete resection.

Only $45 \%$ and $53 \%$ correctly arranged the steps of EMR and identified the optimal electrosurgery setting for EMR, respectively. Knowledge deficiencies in assessment of adenoma morphology by Paris and NICE classification were also identified (48\% correctly identified flat lesions by Paris classification and
- Table 2 Knowledge of in-training fellows in four domains of EMR of large colon polyps.

\section{Domain}

Correct

answers

(\%)

\begin{tabular}{|c|c|}
\hline \multicolumn{2}{|l|}{ Endoscopic assessment of large adenoma } \\
\hline \multicolumn{2}{|l|}{ - 1. Polyp morphology by Paris classification } \\
\hline - Item 16 Identify lesion as Paris 0-Ip & $138(84.7)$ \\
\hline - Item 17 Identify lesion as Paris 0 -Is or 0 -IIa & $117(71.8)$ \\
\hline - Item 18 Identify lesion as Paris 0 -IIb & $79(48.5)$ \\
\hline \multicolumn{2}{|l|}{ - 2. Polyp surface by NICE classification } \\
\hline - Item 19 Identify NICE II lesion & $129(79.1)$ \\
\hline - Item 20 Identify NICE I lesion & $114(69.9)$ \\
\hline - Item 21 Identify NICE III lesion & $138(84.7)$ \\
\hline \multicolumn{2}{|l|}{ - 3. Polyp surface by granularity } \\
\hline $\begin{array}{l}\text { - Item } 22 \text { Identify lesion with higher likelihood } \\
\text { of harboring high-grade dysplasia or invasive } \\
\text { adenocarcinoma }\end{array}$ & $132(81)$ \\
\hline
\end{tabular}

Removal of large adenoma with EMR

- Item 23 Arrange EMR steps in correct order $73(44.8)$

a. Examine polyp $>$ Lift $>$ Resect $>$ Inspect resection site $>$ manage complications $>$ retrieve tissue

b. Examine polyp $>$ Lift $>$ Resect $>$ Inspect resection site $>$ retrieve tissue $>$ manage complications

- Item 24 Recurrence of adenoma with piecemeal vs. en-bloc resection

- Item 25 Management of residual polyp tissue

- Item 26 Thermal therapy of polypectomy edge to $144(88.9)$ reduce adenoma recurrence

- Item 27 Lifting adenoma with submucosal injection

- Item 30 Current used for EMR

- Item 34 Site for tattoo placement $149(91.4)$

Complications of EMR

- Item 28 Most common immediate complication of EMR

- Item 29 Delayed complications of EMR $147(90.2)$

- Item 31 Current for thermal therapy of post-poly$121(74.2)$ pectomy bleeding

- Item 32 Signs of perforation after EMR

Follow-up after resection of large adenomas

- Item 33 Follow-up endoscopy after piecemeal $153(93.9)$ EMR

EMR, endoscopic mucosal resection. 
- Table 3 Factors associated with higher tertiles ordinal regression.

\begin{tabular}{|l|l|l|}
\hline Variable & Odds ratio $\mathbf{( 9 5 \% ~ C l )}$ & P value \\
\hline $\begin{array}{l}\text { Third-year fellow vs. } \\
\text { first-year fellow }\end{array}$ & $10.31(2.21-47.99)$ & 0.01 \\
\hline $\begin{array}{l}\text { Second-year fellow vs. } \\
\text { first-year fellow }\end{array}$ & $5.05(1.14-22.42)$ & 0.01 \\
\hline $\begin{array}{l}\text { Third-year fellow vs. } \\
\text { second-year fellow }\end{array}$ & $2.04(1.06-3.93)$ & 0.001 \\
\hline \begin{tabular}{l} 
Male sex \\
\hline Advanced rotation
\end{tabular} & $3.14(1.48-6.68)$ & 0.01 \\
\hline $\begin{array}{l}\text { Median confidence } \\
\text { Area under the curve: } 72.5 \% .\end{array}$ & $0.67(0.47-0.96)$ & 0.02 \\
\hline \begin{tabular}{l} 
Cl, confidence interval. \\
\hline
\end{tabular}
\end{tabular}

$69.9 \%$ correctly identified hyperplastic lesions by NICE classification). These findings may have important clinical implications. While correct morphologic identification is not reflective of ADR, these clinical skills are beneficial in determining the need for and approach to polyp resection [8, 9]. While uncommon, flat lesions have an increased risk of progressing to malignancy. In addition, detection and complete removal of right-sided sessile lesions has been identified as an important focus of colonoscopy quality initiatives. Fellowship programs should consider providing structured training in polyp morphology assessment as part of a comprehensive EMR curriculum.

Low knowledge scores corresponded with low education scores for assessing adenoma pit pattern, shape, and borders ( $n=91,55.8 \%$; $>$ Fig. 1), knowing the electrosurgery unit settings for EMR ( $n=87,53.4 \%$; Fig. 1 ), and provision of formal education about EMR in the training program $(n=78,47.9 \%$; Item 12). Similarly, low knowledge scores corresponded with low median confidence scores in using digital chromoendoscopy to identify adenomas, knowing electrosurgery unit settings for EMR, and for performing EMR ( $\bullet$ Fig. 2). Median confidence was found to be inversely associated with knowledge of EMR and an increase in median confidence was associated with decreased odds of higher knowledge score (OR $0.67,95 \% \mathrm{Cl}$ : $0.47-0.96)$. Although the finding may be counterintuitive, one possible explanation is that fellows do not perform EMR independently; therefore, their confidence in performing EMR may be higher in relation to the knowledge of EMR. Overall, the results of this study provide insights into the areas of EMR in which fellows have the greatest knowledge deficits. The incorporation of standardized formal training in gastroenterology fellowship with a focus on key areas presents an important educational opportunity to improve fellow knowledge and EMR skills [7, 9-12].

Progression through fellowship was associated with increase in knowledge of EMR,. This finding is expected as fellows get more exposure to observe and perform EMR as they progress through fellowship $[13,14]$. We identified that scheduled rotations in advanced endoscopy during gastroenterology fellowship increased knowledge of EMR (OR 2.25, $95 \%$ Cl: 1.14-
4.44). Advanced endoscopy rotations can provide the opportunity to perform additional EMR cases with experienced physicians. The effect of advanced endoscopy rotations was noted to be independent of the postgraduate year (PGY) level, which highlights the added value of these rotations during fellowship in learning about EMR. Fellow training in EMR may benefit from gastroenterology fellowship program evaluation of the proportion of EMR that is performed by advanced vs non-advanced endoscopy faculty to determine optimal rotational exposures to EMR procedures. Further studies are needed to assess the broader impact of advanced endoscopy rotations during gastroenterology fellowship on procedure skills of gastroenterology fellows.

Male sex was found to have an apparent effect on education score (OR 3.14, $95 \% \mathrm{Cl}: 1.48-6.68)$. It was noticeable that a lower proportion of women participated in this survey study $(27 \%)$ than the proportion of women in gastroenterology fellowships in the United States (32-39\% [15]). EMR of large colon adenomas may be considered an advanced endoscopy skill, which may be associated with a lower interest among women gastroenterology fellows in performing such procedures [16]. While this study was not designed to assess systematic issues related to sex-based differences in training opportunities or knowledge about EMR, further studies should be considered.

This study has several strengths. It is the first of its kind to evaluate the knowledge of gastroenterology fellows in the United States regarding adenoma classification and EMR techniques. Particpants were drawn from a national sample from all the gastroenterology fellowship programs in the United States. In addition, the survey questions were non-ambiguous, developed based on the EMR guidelines issued by the ASGE and ESGE, and approved by ACG's research committee. The survey was comprehensive in collecting data and had a robust statistical methodology to analyze the data as indicated by a high AUC (72.5\%).

There are several limitations to this study. First, the response rate to the survey was low despite our best efforts to administer the survey. The survey administration had to be paused due to the COVID-19 pandemic, which could have affected the response rate. There could have been responses that reflected completion of the survey primarily by the fellows who had exposure to or interest in EMR of large colon polyps. However, a response bias would nean that increased fellow participation would show a higher knowledge deficit. Second, the articipants were predominantly men, second- and third-year fellows, and were from large university programs; therefore, they may not be representative of all the gastroenterology fellows in the country. Even with limited first-year fellow participation, the results show the effect of progression through fellowship on the overall knowledge of EMR and these results are unlikely to change with greater participation by first-year fellows. In addition, greater inclusion of second- and third-year fellows is more likely to represent the training experience of gastroenterology fellowship. Similarly, program setting (community vs. university) was not found to affect the knowledge score and a higher participation from community-based programs is less likely to change the results. 


\section{Conclusions}

In conclusion, we found that nearly half the fellows had no formal education and the survey identified prominent knowledge deficiencies in EMR. We identified that educational efforts should emphasize on overview of the skills, techniques needed to perform EMR including electrosurgery unit settings, and assessment of polyp morphology. Incorporation of standardized formal training with the inclusion of participation in advanced endoscopy rotations could be a key strategy to enhance EMR skills among gastroenterology fellows.

Competing interests

The authors declare that they have no conflicts of interest.

\section{References}

[1] Siegel RL, Miller KD, Jemal A. Cancer statistics, 2020. CA Cancer J Clin 2020; 70: 7-30

[2] Strum WB. Colorectal adenomas. N Engl J Med 2016; 374: 1065-1075

[3] Zauber AG, Winawer S], O'Brien M] et al. Colonoscopic polypectomy and long-term prevention of colorectal-cancer deaths. N Engl J Med 2012; 366: 687-696

[4] Corley DA, Jensen CD, Marks AR et al. Adenoma detection rate and risk of colorectal cancer and death. N Engl J Med 2014; 370: 12981306

[5] Thosani N, Guha S, Singh H. Colonoscopy and colorectal cancer incidence and mortality. Gastroenterol Clin North Am 2013; 42: 619-637
[6] Brenner H, Chang-Claude J, Seiler CM et al. Protection from colorectal cancer after colonoscopy: A population-based, case-control study. Ann Intern Med 2011; 154: 22-30

[7] Hwang JH, Konda V, Abu Dayyeh BK et al. Endoscopic mucosal resection. Gastrointest Endosc 2015; 82: 215-226

[8] Ferlitsch M, Moss A, Hassan C et al. Colorectal polypectomy and endoscopic mucosal resection (EMR): European Society of Gastrointestinal Endoscopy (ESGE) Clinical Guideline. Endosc 2017; 49: 270297

[9] Hwang JH, Konda V, Abu Dayyeh BK et al. Endoscopic mucosal resection. Gastrointest Endosc 2015; 82: 215-226

[10] The Paris endoscopic classification of superficial neoplastic lesions: Esophagus, stomach, and colon - November 30 to December 1, 2002. Gastrointest Endosc 2003; 58: 3-43

[11] Hewett DG, Kaltenbach T, Sano Y et al. Validation of a simple classification system for endoscopic diagnosis of small colorectal polyps using narrow-band imaging. Gastroenterology 2012; 143: 599-607. e1

[12] Tokar JL, Barth BA, Banerjee $S$ et al. Electrosurgical generators. Gastrointest Endosc 2013; 787: 197-208

[13] Miller AT, Sedlack RE, Coyle W] et al. Competency in esophagogastroduodenoscopy: a validated tool for assessment and generalizable benchmarks for gastroenterology fellows. Gastrointest Endosc 2019; 90: 613-620.e1

[14] Sedlack RE. The Mayo Colonoscopy Skills Assessment Tool: Validation of a unique instrument to assess colonoscopy skills in trainees. Gastrointest Endosc 2010; 72: 1125-1133.e3

[15] https://www.abim.org/about/statistics-data/resident-fellowworkforce-data/first-year-fellows-by-gender-type-of-medicalschool-attended.aspx

[16] Pollack M], Cummings LC, Wong RCK et al. Gender disparities and gastroenterology trainee attitudes toward advanced endoscopic training. Gastrointest Endosc 2010; 72: 1111 